\title{
Epstein-Barr virus uveitis after intravitreal triamcinolone injection
}

\author{
Jessica Ong, Josephine C. Richards
}

Department of Ophthalmology, Royal Perth Hospital, Perth, Australia

\begin{abstract}
Purpose: To report a case of Epstein-Barr virus (EBV) uveitis after intravitreal triamcinolone injection.

Methods: Observational case report.

Results: A 66-year-old male presented with bilateral intermediate uveitis, left macular branch retinal vein occlusion, and left macular edema 3 months following acute infectious mononucleosis. He received systemic prednisolone, methotrexate, and intravitreal bevacizumab with partial response. Intravitreal triamcinolone was given for recurrent macular edema, which led to the development of severe panuveitis with positive EBV PCR in aqueous humour. This was successfully treated with high-dose systemic valaciclovir and topical prednisolone.

Conclusion: Non-infectious uveitis may become infectious following intravitreal steroid administration triggering intraocular viral replication. Intraocular fluid should be tested in cases which are suspicious for infection and EBV should be considered a differential diagnosis, particularly if $P C R$ is negative for more common viral etiologies.
\end{abstract}

Keywords: Epstein-Barr virus, panuveitis, uveitis, triamcinolone

\section{Introduction}

Epstein-Barr virus (EBV), a ubiquitous herpes virus associated with infectious mononucleosis, may uncommonly affect any part of the eye during acute systemic infection. In chronic ocular inflammation, however, its PCR presence in ocular fluid specimens is usually regarded as contamination through its dormant presence in resident lymphocytes. We have identified four reports of likely intraocular EBV infection supported by positive EBV PCR in aqueous humour. ${ }^{1,2}$ We present a case of probable intraocular EBV infection resulting in uveitis after intravitreal triamcinolone injection.

Correspondence: Jessica Ong, MBBS(Hons), Department of Ophthalmology, Royal Perth Hospital, 197 Wellington St, Perth, WA 6000, Australia

E-mail: Jessica.Ong@health.wa.gov.au 


\section{Case presentation}

A 66-year-old obese male with metabolic syndrome developed acute infectious mononucleosis confirmed by positive EBV VCA IgG and IgM with atypical monocytosis. EBNA IgG was negative.

Acute infection was followed by chronic fatigue which was treated with oral prednisolone. Within 3 months, he developed a left macular branch retinal vein occlusion with macular edema followed by onset of bilateral floaters. Investigation for common causes of intermediate uveitis was negative. Increased prednisolone dose resulted in decreased vitritis but this recurred once the dose was tapered below $20 \mathrm{mg}$ daily. Methotrexate was used as a steroid-sparing agent. The macular edema and fatigue were controlled but the vitritis never fully resolved. He had bilateral uncomplicated cataract surgery with intraoperative bevacizumab to treat mild left macular edema. After 30 months, methotrexate was ceased permanently because of recurrent respiratory tract infections and impending orthopedic surgery.

Off methotrexate, the vitritis paradoxically decreased but the left macular edema recurred. Due to the concern of a possible systemic viral etiology, the macular oedema was treated locally with left sub-Tenon's triamcinolone $40 \mathrm{mg}$. There was a short-lived improvement in the macular edema with no change in the vitritis. Left intravitreal triamcinolone $4 \mathrm{mg}$ resulted in remission of the macular edema lasting a year with minimal change in the vitritis. A year later, increasing macular edema became visually significant and intravitreal triamcinolone $4 \mathrm{mg}$ was repeated.

Two months later, the patient presented with loss of vision and severe anterior and posterior uveitis. There were dense clusters of tiny white (non-stellate) inferior keratic precipitates. Intraocular pressure was $45 \mathrm{mmHg}$ and visibility was too poor to image the macula for edema. An anterior chamber tap was performed. PCR was positive for EBV and negative for herpes simplex virus 1, herpes simplex virus 2 , and varicella-zoster virus.

A diagnosis of EBV panuveitis was thought likely and the patient was started on valaciclovir $1 \mathrm{~g}$ TDS with intensive topical prednisolone-acetate/phenylephrine-hydrochloride and full ocular hypotensive therapy excluding prostaglandin drops. Within 2 weeks, the anterior uveitis had settled. The ocular hypotensives and topical steroids were tapered and the vitritis gradually improved over 3 months. Optical coherence tomography confirmed absence of macular edema. Interestingly, the microaneurysms previously associated with the vein occlusion became almost undetectable.

More than a year later, the patient developed left steroid-induced, progressive, uveitic glaucoma in the left eye despite maximal tolerable intraocular pressure-lowering therapy. He was on topical steroid treatment with no antiviral 
therapy or systemic immunosuppression. At the time there was mild vitritis with minimal anterior uveitis and no macular edema. During XEN trabeculectomy surgery, an anterior chamber tap was PCR negative for all previously tested pathogens, including EBV.

\section{Discussion}

In this case, the strongly positive PCR result, absence of other herpes viruses, and good response to valaciclovir all strongly suggest intraocular EBV infection and viral replication. Pathogens isolated by metagenomic deep sequencing in uveitic eyes highlight the eye's potential to act as an infectious reservoir. ${ }^{3}$ Intraocular infection may occur through direct invasion of ocular tissue or through infected B-cells which enter the eye during inflammation.' Systemic immunity was apparently strong enough to prevent fulminant infection during treatment with oral steroids, methotrexate, and sub-Tenon's triamcinolone, but the ongoing chronic intraocular inflammation may have been a marker of some viral activity. On this occasion, intravitreal triamcinolone appears to have weakened ocular defenses sufficiently to allow significant EBV activation.

The authors acknowledge that the current literature does not support a high prevalence of EBV uveitis. Sampling of aqueous humor of uveitic eyes revealed a low PCR yield for EBV (1\%) in a Netherlands study of 297 patients. ${ }^{4}$ However, concurrent positive results for other viruses, most commonly varicella-zoster virus, were often found in patients with positive EBV in aqueous humor. ${ }^{4}$ This was one of the study's findings, leading the authors to conclude that intraocular assessment for EBV in uveitis patients has limited value. Our patient had negative PCR for other viruses. Fourteen patients had results indicating only EBV infection with no alternative explanation for uveitis for six patients. ${ }^{4}$ Clinical signs of these patients included anterior chamber inflammation with small KPs and severe vitritis with no involvement of the retina or choroid. All six patients had a chronic, recurrent course of inflammation. The clinical findings described in these patients with potential EBV uveitis are similar to the signs observed in our case.

Several studies demonstrate a reduction of viral replication with acyclovir therapy, the commonest antiviral treatment for ocular EBV infection. ${ }^{5}$ Keorochana reported a case of EBV-associated retinal vasculitis successfully treated with 14 days of intravenous acyclovir, 3 months of oral acyclovir, and vitrectomy. ${ }^{5}$ We found high-dose valaciclovir to be effective in our patient. Intravitreal foscarnet inhibits EBV DNA polymerase and would also be an option for future flares.

Next generation sequencing techniques identifying pathogens in intraocular fluid from patients with uveitis are increasingly demonstrating that intermediate uveitis is not always non-infectious. ${ }^{3}$ Treatment of acute infectious mononucleosis 
with systemic steroids may result in prolonged viral activity. It is possible that the intermediate uveitis in this patient was viral from the start.

Our case is an example of the potential role of steroids in reactivation of EBV from latency, which has been demonstrated in the literature. ${ }^{6}$ Possible mechanisms may include direct promotion of viral replication or inhibition of memory T-cell control of latent virus. ${ }^{6}$

This case illustrates that even when there has been an initial partial favorable response to immunomodulation, a tipping point may be reached at which viral replication may be triggered and non-infectious uveitis becomes infection. Prior to adding immunosuppression to recalcitrant cases or those where treatment is followed by deterioration, intraocular fluid should be tested for infectious causes.

\section{Declarations}

\section{Consent for publication}

The patient provided informed consent for the use of the clinical information contained in this case report.

\section{Competing interests}

None to declare.

\section{Funding}

None to declare.

\section{Acknowledgements}

The authors wish to thank Dr. Chris Kennedy, consultant vitreoretinal surgeon, for his involvement in the management of this case.

\section{References}

1. Heiligenhaus A, Dohrmann J, Koch J, et al. Severe bilateral panuveitis in a patient with asymptomatic Epstein-Barr virus infection. Eye. 2001;15(6):792.

2. Usui M, Sakai J. Three cases of EB virus-associated uveitis. Int Opthalmol. 1990;14:371-376.

3. Doan T, Wilson MR, Crawford E, et al. Illuminating uveitis: metagenomic deep sequencing identifies common and rare pathogens. Genome Med. 2016;8(1):90-98.

4. Groen-Hakan F, Van Der Eijk AA, Rothova A. The Usefulness of Aqueous Fluid Analysis for EpsteinBarr Virus in Patients with Uveitis. Ocul Immunol Inflamm. 2018. https://doi.org/10.1080/092739 48.2018.1543709.

5. Keorochana N. A case report of Epstein-Barr virus-associated retinal vasculitis: successful treatment using only acyclovir therapy. Int Med Case Rep J. 2016;9:213-218.

6. Cacopardo B, Nunnari G, Mighini MT, et al. Fatal hepatitis during Epstein-Barr virus reactivation.

Eur Rev Med Pharmacol Sci. 2003;7:107-109. 